Nippon Suisan Gakkaishi $\quad \mathbf{5 3 ( 3 )}, 381-383(1987)$

\title{
Equations for the Weight in Air of Nylon Netting*1
}

\author{
Tadanobu Machii*2 and Yukio Nose*3
}

(Accepted July, 15, 1986)

\begin{abstract}
The equations of netting weight in air are very important in designing fishing gears. We developed some equations of English knot nylon netting weight in air for a single mesh ( $\omega$ : weight of two legs and one knot) and for one strip of netting ( $W$ : weight of a 100 fathom length with a depth of 100 meshes), The latter derived from the equations of a single mesh. In order to determine the constants of the equations, we used the Twine Weight Table and measured different factors of netting. Weight $(W)$ is represented by both the number of yarns $(n)$ and mesh size $\left(L_{m}\right)$, as $W=$ $C_{1} \cdot n+C_{2} \cdot\left(n^{3 / 2} / L_{m}\right)$, where $C_{1}$ and $C_{2}$ are the constants. Using the constants determined by the measurements, $\omega$ and $W$ can be written respectively as follows; $\omega=\pi \cdot(D / 2)^{2}(L+9 \cdot D) \cdot \rho$ and $W=$ $n\left\{1+\left(\sqrt{n} / 3 \cdot L_{m}\right)\right\}$, where $D$ is the diameter of twine, $L$ is the length of one leg, and $\rho$ is the density of twine.
\end{abstract}

According to Honda, it is difficult to determine the weight of the netting based on equations, but these are very necessary for the calculations required for designing fishing gears.

Nagamine $^{2 y}$ and Miyamoto ${ }^{3\rangle}$ studied the netting weight in the air for natural fibers, while Iitaka") carried out a similar study in the water for synthetic fibers. They dealt with English (or Weaver's) knot and with Japanese one strip of netting (100 ken $^{* 4}$ long and 100 meshes deep). Other studies related to this subject are unknown to us.

The purpose of this study is two fold: first, to develop equations of weight in the air for a single mesh (as minimum fundamental unit), and for one strip of netting derived from the single mesh equations. Second, based on calculations and measurements, to determine the constants that appear in the equations mentioned above.

\section{Materials and Methods}

\section{Materials}

In order to determine the constants, we used the Twine Weight Table and materials of nylon netting (English knot, multifilament, 210 denier and three strand twine from 6 to 180 yarns).

\section{Methods}

Definitions in this study are as follows; A single mesh consists of two legs and one knot. Mesh size is the length of two legs and two knots, and one strip of netting is equivalent to 100 fathoms' length with a depth of 100 meshes. Fig. 1 shows the definitions of lengths for a single mesh and one strip of netting. The density of the nylon twine $(\rho)$ is $1.14\left(\mathrm{~g} / \mathrm{cm}^{3}\right)$.

The weight per length of twine $(\eta)(\mathrm{g} / \mathrm{cm})$ and the diameter of twine $(D)$ for a given number of yarns $(n)$ are taken from the Twine Weight Table ${ }^{5)}$ and mesh size $\left(L_{m}\right)$ is a known variable.

Unknown variables are the length of one leg $(L)$, the length of one knot $\left(L_{k}\right)$ and the length of twine to make one knot $(l)$. These were determined by measurements.

Using a sample of netting which contained about 100 knots for each $n$, we measured all $L$ and the weight $\left(W_{t}\right)$, and counted the total number of legs $\left(n_{l}\right)$ and knots $\left(n_{k}\right)$. From measurements, we are able to obtain the mean value of $L$, therefore, $L_{k}$ and $l$ are calculated respectively as follows:

$$
L_{k}=\frac{L_{m}}{2}-L
$$

and

*1 This paper was presented at the annual meeting of the Japanese Society of Scientific Fisheries, Sendai, Japan, October, 1984.

*2 Department of Fishing Science \& Technology, Tokyo University of Fisheries, Konan 4-5-7, Minato,

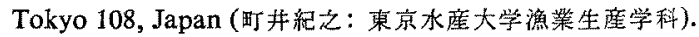

*3 Department of Fisheries, Faculty of Agriculture, The University of Tokyo, Yayoi 1-1-1, Bunkyo, Tokyo 113, Japan (能势幸雄: 東京大学農学部水鷹学科).

*4 $100 \mathrm{ken}=151.5 \mathrm{~m}$, Japanese traditional fishing unit. 


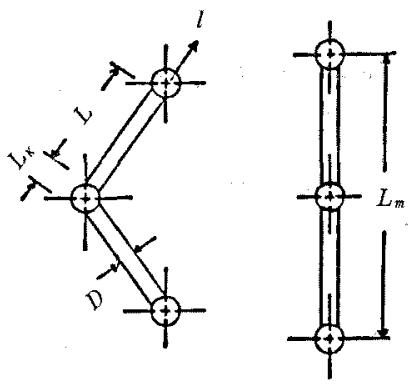

A single mesh of netting

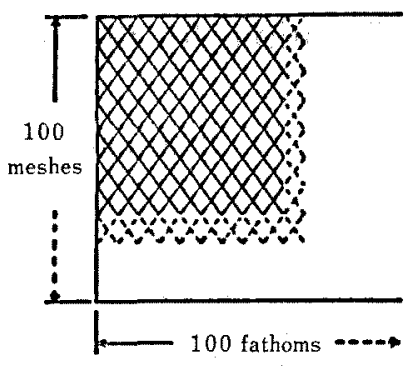

One strip of netting

Fig. 1. Definitions of lengths for a single mesh and one strip of netting. $L$ : Length of one leg, $L_{k}$ : Length of one knot, $l$ : Length of twine to make one knot, $D:$ Diameter of twine, and $L_{\mathrm{m}}:$ Mesh size.

$$
l=\frac{1}{n_{k}}\left(\frac{W_{t}}{\eta}-L \cdot n_{l}\right)
$$

\section{Equations}

The units used are grams for weight of a single mesh and kilograms for weight of one strip of netting, and centimeters relating to lengths of netting.

Weight in the air for a single mesh $(\omega)$

1) $\omega$ expressed by $L$ and $D$;

$\eta$, from it's definition, is written as

$$
\eta=k_{1} \cdot \pi \cdot\left(\frac{D}{2}\right)^{2} \cdot \rho
$$

where $k_{1}$ is a constant related to the weight per length.

Since $l$ is proportional to $D$, it follows that

$$
l=k_{2} \cdot D
$$

where $k_{2}$ is a constant.

From the equations (3) and (4), $\omega$ can be expressed as follows:

$$
\begin{gathered}
\omega=K_{1} \cdot 2 \pi \cdot\left(\frac{D}{2}\right)^{2} \cdot L+K_{2} \cdot D^{3} \\
\left(K_{1}=k_{1} \cdot \rho\right) \\
\left(K_{2}=K_{1} \cdot \frac{\pi}{4} \cdot k_{2}\right)
\end{gathered}
$$

2) $\omega$ expressed by $L_{m}$ and $D$;

Since $L_{k}$ is proportional to $D$

$$
L_{k}=k_{3} \cdot D
$$

where $k_{3}$ is a constant.

Substituting the equations (1) and (6) into the equation (5), we obtain the second form of $\omega$ as :

$$
\begin{array}{r}
\omega=K_{1} \cdot \pi \cdot\left(\frac{D}{2}\right)^{2} \cdot L_{m}+K_{3} \cdot D^{3} \\
\left(K_{3}=K_{2}-K_{1} \cdot \frac{\pi}{2} \cdot k_{3}\right)
\end{array}
$$

3) $\omega$ expressed by $L_{m}$ and $n$;

$\eta$, from the other definition, is given by

$$
\eta=k \cdot d \cdot n
$$

where $k$ is a constant relating to the weight per length.

Determining $D$ from the equations (3) and (8),

$$
D=k_{4} \cdot \sqrt{n}
$$

where $k_{4}$ is a constant which include $k$.

Substituting the equation (9) into the equation (7), the third form of $\omega$ is obtained as:

$$
\begin{gathered}
\omega=K_{4} \cdot n \cdot L_{m}+K_{6} \cdot n^{3 / 2} \\
\left(K_{4}=K_{1} \cdot \frac{\pi}{4} \cdot k_{4}{ }^{2}\right) \\
\left(K_{5}=K_{8} \cdot k_{4}^{3}\right)
\end{gathered}
$$

As mentioned above, weight in the air relating to a single mesh $(\omega)$ can be represented by different terms as the equations (5), (7) and (10).

Weight in the air for one strip of netting (W) $W$ expressed by $L_{m}$ and $n$;

The total number of mesh included within one strip of netting $(M)$ can be written as

$$
M=\frac{c}{L_{m}}
$$

where $c$ is a constant.

Consequently, multiplying the equation (10) by the equation (11), $W$ can be expressed as

$$
W=C_{1} \cdot n+C_{2} \cdot\left(\frac{n^{3 / 2}}{L_{m}}\right)
$$


Table 1. Constants obtained from the Twine Weight Table and from the measurements of the netting

\begin{tabular}{rcccc}
\hline$n$ & $k_{1}$ & $k_{2}$ & $k_{3}$ & $k_{4}$ \\
\hline 6 & 0.465 & 20.0 & 2.40 & 0.0245 \\
9 & 0.482 & 19.9 & 2.18 & 0.0243 \\
12 & 0.479 & 18.8 & 2.05 & 0.0245 \\
15 & 0.485 & 17.9 & 2.00 & 0.0245 \\
18 & 0.490 & 17.3 & 1.97 & 0.0245 \\
21 & 0.496 & 17.4 & 1.96 & 0.0244 \\
24 & 0.496 & 17.5 & 1.96 & 0.0245 \\
27 & 0.494 & 17.2 & 1.95 & 0.0246 \\
30 & 0.497 & 17.8 & 1.97 & 0.0247 \\
36 & 0.499 & 17.6 & 2.00 & 0.0247 \\
45 & 0.501 & 17.8 & 2.06 & 0.0248 \\
51 & 0.502 & 18.6 & 2.10 & 0.0248 \\
60 & 0.506 & 18.2 & 2.18 & 0.0248 \\
75 & 0.505 & 17.6 & 2.28 & 0.0249 \\
90 & 0.513 & 17.8 & 2.37 & 0.0249 \\
120 & 0.513 & 16.4 & 2.48 & 0.0250 \\
150 & 0.519 & 16.9 & 2.49 & 0.0251 \\
180 & 0.521 & 16.9 & 2.49 & 0.0251 \\
\hline Mean & 0.498 & 17.9 & 2.16 & 0.0247 \\
\hline
\end{tabular}

Table 2. Constants calculated by the mean values of constants shown in Table 1

\begin{tabular}{llll}
\hline \multicolumn{2}{c}{ A single mesh of netting*1 } & One strip of netting*2 \\
\hline$K_{1}$ & 0.568 & $C_{1}$ & 0.995 \\
$K_{2}$ & 7.97 & $C_{2}$ & 0.333 \\
$K_{3}$ & 6.04 & & \\
$K_{4}$ & $2.72 \times 10^{-4}$ & \\
& $K_{5}$ & $9.10 \times 10^{-3}$ & \\
\hline$* 1$ & A single mesh of netting consists of two legs and one knot. \\
$* 2$ & One strip of netting is equivalent to 100 fathoms' long and \\
& 100 meshes deep.
\end{tabular}

$$
\begin{aligned}
& \left(C_{1}=K_{4} \cdot c \times 10^{-3}\right) \\
& \left(C_{2}=K_{5} \cdot c \times 10^{-3}\right)
\end{aligned}
$$

As mentioned above, weight in the air for one strip of netting $(W)$ can be presented by practical form of the equation (12) in terms of $L_{m}$ and $n$.

\section{Results and Discussion}

Table 1 shows the constants obtained from the Twine Weight Table and from the measurements of the netting.

The constants $k_{1}$ and $k_{4}$, from the data given by the Twine Weight Table, were obtained by calculations of the equations (3) and (9) respectively. Other constants $k_{2}$ and $k_{3}$ were determined by calculations of the equations (4) and (6) based on substitution of the data given by the measurements in the equations (1) and (2).

Considering that all constants for each $n$ are nearly uniform, the mean values of the constants are shown in the lowest part of Table 1.

Using these mean values, the new constants that appear in the equations of weight in the air are calculated for both, a single mesh and one strip of netting. The results are shown in Table 2 .

The values of $c$ can be determined from the definition of one strip of netting. Since $M$ is equivalent to twice the lateral number of mesh within 100 fathoms $\left(182.9 \times 10^{2} / L_{m}\right)$ by longitudinal number of mesh (100),

$$
c=3.658 \times 10^{\circ}
$$

In conclusion, for a single mesh and for one strip of netting we derived the equations of weight in the air as (5), (7), (10) and (12). From the results obtained, we can show two representative equations as follows. $\omega$ for a single mesh of netting;

$$
\omega=\pi \cdot\left(\frac{D}{2}\right)^{2} \cdot(L+9 \cdot D) \cdot \rho
$$

$W$ for one strip of netting;

$$
\begin{gathered}
W=n \cdot\left(1+\frac{\sqrt{n}}{3 \cdot L_{m}}\right) \\
(6 \leqq n \leqq 180)
\end{gathered}
$$

In this study, we could not compare the results with other studies related to the weight of a single mesh and one strip of netting. Such comparisons will have to be done in a future study.

\section{References}

1) K. Honda: "Gyogu Zairyō" (Fishing Gear Materials), Kōseisha kōseikaku, Tokyo, 1981, pp. 41 .

2) T. Nagamine: "Saishin Gyorōgaku" (Modern Fishing Arts), Kōseikaku, Tokyo, 1932, pp. 4345.

3) H. Miyamoto: "Teichiami Gyoron" (Stationary Fishing Gears), Kawade shobö, Tokyo, 1954, pp. 93-96.

4) Y. Iitaka: Nippon Suisan Gakkaishi, 24, 620-621 (1958).

5) Twine Weight Table: "Yoriito Kikakuhyō", Nihon Gyomō Sengu, Tokyo, 1966, pp. 8-9. 